Recherches en didactique des langues et des cultures

Les cahiers de l'Acedle

13-1 | 2016

Interactions langagières et didactique des langues

\title{
Faciliter l'acculturation à l'écriture universitaire des étudiants de licence de français en Algérie : genres discursifs et rapport à l'écrit
}

M'Hand Ammouden et Claude Cortier

\section{OpenEdition}

Édition électronique

URL : http://journals.openedition.org/rdlc/900

DOI : $10.4000 /$ rdlc. 900

ISSN : 1958-5772

Éditeur

ACEDLE

Référence électronique

M'Hand Ammouden et Claude Cortier, «Faciliter l'acculturation à l'écriture universitaire des étudiants de licence de français en Algérie : genres discursifs et rapport à l'écrit », Recherches en didactique des langues et des cultures [En ligne], 13-1 | 2016, mis en ligne le 12 octobre 2016, consulté le 30 avril 2019. URL : http://journals.openedition.org/rdlc/900 ; DOI : 10.4000/rdlc.900

Ce document a été généré automatiquement le 30 avril 2019

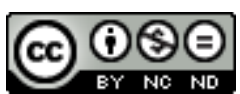

Recherches en didactique des langues et des cultures is licensed under a Creative Commons AttributionNonCommercial-NoDerivatives 4.0 International License 


\title{
Faciliter l'acculturation à l'écriture universitaire des étudiants de licence de français en Algérie : genres discursifs et rapport à l'écrit
}

\author{
M'Hand Ammouden et Claude Cortier
}

Dans la plupart des systèmes éducatifs, le passage d'un palier à l'autre de l'enseignement et notamment de l'enseignement secondaire à l'université pose problème, il entraine généralement plusieurs ruptures simultanées » et s'accompagne, selon Coulon (2005:4), d'importants changements dans les rapports qu'entretient l'étudiant avec des « modalités fortement présentes dans tout apprentissage : d'une part avec le temps, d'autre part avec l'espace, [...] mais également par rapport aux règles et aux savoirs». Précisons que, dans le contexte algérien, la rupture n'est pas seulement pédagogique, "méthodologique et discursive " (Pollet, 2001) : pour les étudiants qui vont suivre des études scientifiques, techniques et technologiques, médicales, elle est aussi linguistique et cognitive dans la mesure où ces étudiants passent d'un enseignement dispensé en arabe à un enseignement dispensé en français (Cortier, Hachadi \&Amar Sharif, 2009). En effet, le français bénéficie aujourd'hui en Algérie d'un statut de langue étrangère privilégiée, il est langue seconde d'une partie de la population et surtout langue d'enseignement dans toutes les filières universitaires scientifiques, médicales, techniques et technologiques voire pour certains modules en sciences économiques et sociales. Or, il n'est enseigné que comme première langue étrangère à partir de la $3^{e}$ année primaire, le volume horaire consacré à son enseignement est très en deçà de ce que prévoient les programmes, et la qualité de l'enseignement très inégale suivant les régions et les milieux sociaux. Dans les départements universitaires de français où les enseignants se refusent à recourir à l'arabe ou à des formes de parlers bilingues comme dans les autres filières, le taux d'échec est très important en première année, il est en moyenne de $50 \%$ mais peut avoisiner les $70 \%$ comme en 2008-2009 à l'université de Blida ${ }^{1}$. 
2 On doit toutefois se demander aujourd'hui si la question linguistique arabe/français, symptomatique d'un rapport aux langues conflictuel, n'a pas tendance à masquer, voire occulter les autres points de difficultés que pose ce passage du secondaire au supérieur. On sait qu'il n'existe pas de facteurs univoques de cet échec mais « une constellation de facteurs intercorrélés» (Maurice, 2001: 78) : la maîtrise de la langue d'enseignement et d'étude, une motivation forte allant de pair avec un projet clairement défini, des méthodes de travail adaptées, un investissement de temps suffisant consacré à l'étude, une perception juste du contexte académique et des attentes des professeurs sont ainsi des facteurs conjugués de réussite (ibid.). La massification de l'enseignement universitaire et la démocratisation de l'accès aux études supérieures font que, depuis quelques années, on se préoccupe de plus en plus de faciliter ce passage. C'est désormais aussi le cas de l'Algérie, dont les volontés de réforme du système éducatif sont affirmées dans l'enseignement supérieur depuis 2004, et où l'on n'hésite pas à parler de "pédagogie universitaire» et de formation des enseignants à l'enseignement supérieur, notamment dans le cadre de la mise en place du système LMD (Licence, Master, Doctorat). C'est pourquoi il nous a semblé utile de développer des réflexions en direction des autres variables de "l'affiliation universitaire» (Coulon, 2005), telles que les motivations pour la filière d'étude et les représentations qui y sont liées mais aussi les méthodologies de travail universitaire, les genres académiques et les formes d'évaluation correspondantes (De Ketele, 2010). Les travaux conduits dans le cadre de la didactique du français dans le supérieur (Fintz, 1998; Dabène et Reuter, 1998; Delcambre \& Jovenet, 2002), de la didactique du français sur objectifs spécifiques et universitaires (Mangiante \& Parpette, 2010, 2011; Cortier \& Kaaboub, 2010) nous ont mis sur la voie d'une didactique des discours universitaires et d'une approche liée aux genres plutôt qu'aux types de texte (Defays, Englebert et al., 2009) pour privilégier le développement de compétences méthodologiques discursives.

3 Nous nous proposons d'examiner dans le cadre de cet article, les possibilités de développer cette approche au sein de l'université algérienne, dans le cadre de la mise en place du LMD, en appui sur des travaux de recherche en cours ${ }^{2}$. Nos recherches visent à relier l'élaboration des curricula et l'enseignement / apprentissage aux profils et aux besoins des étudiants de licence de français, par le biais d'enquêtes sur les programmes et les cours associés à des tests de niveaux. Elles entendent également faire émerger et analyser leurs représentations dominantes face aux apprentissages et aux formes de littéracies, universitaires ou non, leurs rapports à l'écrit (Barré de Miniac, 2000) et les caractéristiques des textes et discours "réussis» grâce au recueil d'un corpus de productions écrites à partir duquel nous avons pu réaliser une analyse de contenu.

4 L'examen des programmes de français pourra nous être utile pour examiner le statut de l'écrit et des textes et le traitement didactique qui leur est accordé. Nous supposons que l'on ne peut parler de littéracie que si les textes et les écrits abordés dans les langues d'enseignement, les langues enseignées ou les langues de l'environnement sont mis en perspective à certains moments du parcours et intégrés dans une même compétence littéraciée ou plurilittéraciée (Moore, 2006) soit simultanément soit successivement. Si la notion de littéracie s'avère fort utile pour penser la continuité des enseignements/ apprentissages (Delcambre, Lahanier-Reuter \& 2010), elle sous-entend en même temps une culture de l'écrit qui ne peut se cantonner dans une vision cloisonnée des langues, des discours, des variétés d'écriture. 


\section{Confrontations et / ou ruptures linguistiques, didactiques et culturelles}

\section{Langues maternelles ou de première socialisation et arabe scolaire}

5 L'Algérie est un pays plurilingue où seul l'arabe classique / standard est langue officielle tandis que les variétés de l'arabe algérien ne sont reconnues ni comme officielles ni comme nationales à la différence de la langue amazighe ou berbère, qui a pu accéder au statut de langue nationale en 2002 mais qui est encore très peu enseignée et dont les variétés sont également utilisées quotidiennement par une partie importante de la population.

6 Selon K. Taleb-Ibrahimi (1997, 2007, 20103), la première rupture à laquelle ont affaire les jeunes Algériens ne se situe pas dans le passage lycée-université. Elle se fait entre la langue de l'école (arabe littéral ou arabe scolaire) et la langue de la maison (arabe dialectal ou variété amazighe). Dans la mesure où les enfants scolarisés sont exposés dès leur jeune âge à un «artefact de langue » et où nulle part on ne prend en compte le ou les arabes algériens, on pourrait parler d'un "échec» de la politique d'arabisation reconnu par de nombreux chercheurs. On enseigne une langue difficile, vieillie, sans lien avec la littérature contemporaine, les textes de la presse ou des media alors que les variétés d'arabe parlé font preuve d'une grande vitalité. Cette rupture est accentuée par une forte tradition grammaticale et écrite de la langue arabe et de son enseignement et l'intégration d'une "surnorme» liée au caractère sacré et prestigieux de la langue classique, langue du Coran. L'opposition langue / dialecte(s) et la dévalorisation systématique de ces derniers contribue à renforcer les phénomènes de diglossie et probablement à inhiber les formes personnelles d'écriture.

\section{Les langues d'enseignement : confrontations et ruptures}

7 En Algérie, les sciences naturelles, médicales, physiques, techniques et les technologies ${ }^{4}$ sont enseignées en français. Plus de la moitié des étudiants qui arrivent à l'université pour suivre des études dans ces filières sont confrontés à une rupture brutale d'ordre linguistique, cognitif et méthodologique qui résulte du passage, sans préparation en amont et parfois même sans information, d'un enseignement en arabe à un enseignement en français (Cortier, Hachadi \& Amar Sharif, 2009).

8 Ils sont de fait confrontés à la situation d'étudier en langue seconde ou étrangère dans leur propre pays mais ne sont-ils pas également confrontés à la coexistence de deux cultures éducatives et didactiques? L'une issue d'une tradition fortement marquée de l'enseignement de l'arabe, centrée sur l'acte d'enseignement et davantage appuyée sur des fondements grammaticaux, rhétoriques et littéraires, l'autre étant celle des enseignements scientifiques dispensés en français de caractère pragmatique ou celle des langues étrangères orientées vers l'apprenant et ses apprentissages et les formes de communication (Ammouden \& Cortier, 2009).

9 Comme cela a pu être souligné récemment ${ }^{5}$, le français et l'arabe offrent cependant des points de similarité, qui peuvent expliquer certains des paradoxes voire des résistances aux réformes rencontrés dans l'enseignement du français en Algérie. Ce sont deux langues historiques, deux « grandes langues » à caractère international, dites supercentrales 
${ }^{6}$ qui, très grammatisées (abondance du patrimoine écrit, grammaires, dictionnaires), entretiennent une relation très forte à l'écrit. Ce sont donc des langues également marquées par un très fort rapport à la Norme. Mais ce sont des langues de grande créativité dans leurs variétés actuelles: elles connaissent des formes d'affirmation diversifiées et se trouvent de la même façon en tension entre créativité extrême et norme / surnorme, dans leur rapport à la pluralité. Ces aspects sont renforcés par les caractéristiques de leurs cultures éducatives et didactiques dominées par les traditions grammaticales, la tradition du Livre sacré pour l'arabe, celle des Belles-Lettres et du « bon usage » pour le français. Cependant, en Algérie, le Français Langue Étrangère (FLE) a fait une entrée didactique dans les années 1980-1990, consécutivement à la création de l'École fondamentale et à la progression de l'arabisation de l'enseignement scolaire ${ }^{7}$. Le français jusque-là enseigné avec les manuels français (dont les célèbres Lagarde \& Michard) devient officiellement une "langue étrangère » comme les autres. D'une part, l'inspection générale s'attelle à l'organisation de la formation des enseignants du secondaire et sollicite des spécialistes à la pointe du FLE (Courtillon, Moirand) pour introduire l'approche notionnelle-fonctionnelle puis l'approche communicative. D'autre part, les premiers accords-programmes sont signés au niveau universitaire notamment entre l'université de Blida et le Crapel (Centre de Recherche et d'Applications Pédagogiques en Langues, Nancy) pour mettre en place la didactique du $\mathrm{FLE}^{8}$. Ces premières mesures seront accompagnées et suivies par une politique active de coopération. La didactique du FLE, français fonctionnel puis Français sur Objectifs Spécifiques (FOS) et les approches communicatives se développeront parallèlement aux travaux français ou francophones. C'est pourquoi l'on a pu conclure à l'existence, dans les représentations sinon dans les faits, de « deux cultures éducatives et didactiques » aujourd'hui en opposition (Ammouden \& Cortier, 2009). Que ce soit dans les rapports aux langues et dans les cultures linguistiques, dans les conceptions de la formation et de l'enseignement / apprentissage (cultures éducatives) ou dans les cultures didactiques (traditions associées à l'enseignement de telle ou telle langue ou discipline), on constate que le français et l'arabe demeurent aujourd'hui dans des perspectives plus parallèles que convergentes, en dépit d'initiatives développées par les concepteurs de programme ${ }^{9}$, lors des premières années d'apprentissage du français, et par les didacticiens du Français Langue Étrangère et Seconde (Fles), pour développer approche communicative puis approche par les compétences. Ceci pourrait expliquer les difficultés et résistances à développer de nouvelles approches, car elles entrent en conflit avec les habitus locaux.

Ces mêmes hypothèses devraient conduire à interroger les acquis antérieurs des étudiants dans les cours de langue arabe, mais aussi dans les matières littéraires enseignées avant l'université en arabe (philosophie, histoire, etc.), qui ne peuvent qu'avoir une influence sur les représentations des étudiants et de là sur l'apprentissage dans les modules de langue, dans une perspective de didactique comparée. Ces éléments nous semblent de nature à freiner l'entrée dans la sphère académique et l'acquisition des discours universitaires dominants à l'université, en l'absence de réflexion et de travail sur les représentations des étudiants face aux exigences académiques et en l'absence de réflexion vers une didactique adaptée ou convergente (Miled, 2005, 2008). 


\section{Quelle(s) continuité(s) et rupture(s) dans l'enseignement du français ?}

\section{Les programmes de français scolaires}

11 Les apprenants algériens qui suivent un cursus scolaire complet jusqu'au bac étudient le français durant dix années. L'horaire accordé au français sur l'ensemble des cycles devrait permettre d'atteindre théoriquement un niveau B2 (selon le Cadre Européen Commun de Référence pour les Langues, Cecrl), au moins dans les compétences privilégiées en milieu scolaire c'est-à-dire l'écrit. Les nouveaux programmes rédigés avec l'appui d'experts européens et en application progressive depuis 2004 mettent l'accent sur l'approche par compétences, les notions de textes et de discours et la dimension communicative.

Les contenus des programmes sont globalement orientés vers la lecture / production de textes rencontrés dans des situations de la vie quotidienne. Les projets et séquences proposées sont tantôt fondés sur la logique des "types de textes" (le narratif, l'argumentatif, l'explicatif, le descriptif), tantôt sur celle des genres textuels (l'interview, le fait divers, le reportage et le récit de voyage, l'appel, mais aussi les affiches prescriptives, notices, dépliants, annonces, menus, cartes d'invitation etc.).

En dépit d'allusions à la linguistique de l'énonciation, à une approche discursive ou par compétences, les références essentielles demeurent l'approche communicative et les techniques d'expression sans lien avec les genres.

La lecture de ces programmes du secondaire montre que l'on souhaiterait associer à chaque projet une ou des technique(s) d'expression écrite ou orale : la prise de notes à partir de l'écrit ou de l'oral, le plan, le résumé, l'exposé oral, la fiche de lecture, l'essai, la synthèse de document, le compte rendu, etc. Cependant, l'examen approfondi des nouveaux manuels publiés amène à constater, dans les propositions faites, un manque de richesse textuelle qui nuit à l'approche technique et parfois de cohérence méthodologique.

On constate l'insertion, dans un même projet, d'intentions de communication ou d'objets d'étude, de séquences et / ou de techniques d'expressions qui ne concourent pas vers la réalisation de ce projet ou qui ne convergent pas vers un genre, un univers ou une formation discursive, ce qui ne favorise guère la mobilisation des savoirs et savoirs-faires ou la cohérence au sein des différents projets pas plus qu'une approche en domaine, univers ou genre discursif. On constate de même la coexistence dans un même programme ( $1^{\text {ère }}$ année secondaire), de séquences didactiques dont l'objet d'étude est un genre textuel, le fait divers, assez peu motivant d'ailleurs pour des adolescents ${ }^{10}$, avec d'autres séquences qui ont pour objet d'étude un type de texte, le discours argumentatifrécit, sans référence à des genres accessibles.

Si les « objets d'étude » des onze projets proposés sont pour la plupart des genres textuels, les séquences proposées sont pour la plupart essentiellement fondées sur la logique des techniques d'expression et les savoir-faire associés. On peut donc retenir la coexistence dans un même programme et / ou cursus de logiques de références qui nous semblent peu compatibles et conclure qu'en définitive seules les techniques d'expression (programmes du secondaire) pourraient préparer à la réception / production des genres 
textuels et discursifs dominants à l'université, à condition d'être réellement pratiquées et surtout d'être associées à des ensembles de textes diversifiés, pour éviter un applicationnisme réducteur.

\section{Les programmes universitaires (unités d'enseignement fondamentales et méthodologiques) : des types aux genres de textes?}

17 Avec l'introduction du système LMD depuis 2005-2006 et la refonte des programmes et curricula universitaires qui s'ensuit, le volume horaire consacré à l'apprentissage de la langue (oral et écrit) en Licence de langue française a été augmenté de façon sensible, d'une part, parce qu'il fallait ramener à trois ans une formation auparavant de quatre ans, d'autre part parce que les universités souhaitent améliorer les compétences en langue des étudiants, en raison des faiblesses constatées et du profil requis en fin de licence. L'écrit enseigné auparavant dans les modules "Pratique Systématique de la Langue " (une séance hebdomadaire en $1^{\text {ière }}$ et $2^{\text {ième }}$ année) et "Techniques de l'Expression Ecrite et Orale 》 (une séance hebdomadaire en $1^{\text {ière }}$ année), qui théoriquement prennent en charge également l'oral, se voit désormais attribuer, à lui seul, trois heures hebdomadaires et s'étaler sur les trois années de la licence.

18 L'étude de trois référentiels mis en œuvre en 2005-2006 dans de grandes universités algériennes ${ }^{11}$ montre une prédominance de la typologie des textes. En témoignent les intitulés des unités didactiques: "l'injonctif», «l'explicatif», "le narratif», "l'argumentatif» ou encore: "Apprentissage des techniques de l'écrit, l'étude des différents types de textes mis à la portée des étudiants: le narratif, descriptif, argumentatif, prescriptif». On trouve cependant, quelques intitulés visant les genres de textes : la fiche de lecture, l'exposé, le commentaire de texte, mais catégorisés avec les techniques d'expression. Témoignent également de cette orientation toujours marquée, une série de manuels réalisés à l'Université d'Oran ${ }^{12}$, à partir de 2007 dont les titres parlent d'eux-mêmes: «Le texte narratif», «Le texte explicatif», "Le texte argumentatif », « Le texte injonctif ».

\section{Qu'en est-il dans les pratiques universitaires ?}

19 Pour réaliser l'inventaire des genres de textes écrits auxquels les étudiants de licence de français ont souvent affaire en situation de réception ou de production, nous avons analysé un corpus constitué de différents polycopiés et sujets d'évaluation remis aux étudiants de la licence, au cours de l'année universitaire 2009 / 2010, dans différents enseignements (plus de 200 pages). Les résultats de cette étude, appuyés par des entretiens avec des étudiants et des enseignants, font apparaître quatre grandes catégories, les deux premières étant les plus largement représentées :

- Les écrits documentaires et didactiques des disciplines enseignées : articles de dictionnaires et d'encyclopédies, extraits d'ouvrages de didactique ou de linguistique, d'histoire littéraire ;

- Les écrits de recherche : pages de mémoires, articles scientifiques, extraits d'ouvrages de didactique et de linguistique ;

- Les écrits littéraires : poèmes, extraits de romans et de pièces de théâtre ;

- Les fiches «techniques d'expression ou de rédaction »: rédaction d'une lettre administrative, d'un CV, d'une dissertation, d'un commentaire composé, etc. 
ace accordée aux écrits de recherche dans certains cursus de licence mérite d'être questionnée, car il est fréquent que les enseignants proposent dès le premier semestre des cours de méthodologie de la recherche à la place de ceux de Méthodologie du Travail Universitaire (désormais MTU), alors que les apprenants ne réalisent aucun mémoire même en fin du cursus de la licence. Les écrits académiques comme genre étudiés apparaissent assez peu, en revanche, ils retrouvent une place de choix dans les activités de production écrite. Seraient ainsi pratiqués des genres variés : l'essai, la dissertation littéraire, le commentaire de citation ou de passages, le commentaire composé, le compte-rendu de lecture et le compte-rendu critique, la fiche de lecture, l'exposé, le résumé, la synthèse de documents.

21 L'unité d'enseignement MTU introduite récemment devrait pouvoir favoriser l'apprentissage des genres de textes auxquels sont confrontés les étudiants dans les cours et évaluations, si l'on considère que sont réellement travaillés les savoir-faire utiles aux différentes modalités de cours et d'examen. Les extraits d'un curriculum universitaire montrent l'affichage, au moins dans les programmes, d'une préparation aux écrits universitaires : prendre des notes et transcrire, réaliser un résumé, réaliser une synthèse, réaliser un compte-rendu, faire un exposé, etc. Ainsi, renforcement linguistique et MTU apparaissent de première nécessité pour l'acculturation universitaire mais ces modules ne doivent pas être conçus séparément. Ils doivent être intégrés dans une didactique plus englobante des discours universitaires où, si le français est la langue cible, les transferts de compétences et de connaissances doivent pouvoir se faire d'une sphère linguistique à l'autre, d'un cursus à l'autre.

Pour conclure sur la question des programmes et des curricula, on ne doit pas dissimuler le fait qu'ils obéissent à une réelle continuité mais dans une orientation, la typologie des textes, désormais contestée, voire réfutée par celui qui en fut l'un des créateurs, J. M. Adam :

En dépit de ce que j'ai pu écrire encore au début des années 1980, sous l'influence des travaux anglo-saxons, pour moi, d'un point de vue épistémologique et théorique, le concept de types de textes est plus un obstacle méthodologique qu'un outil heuristique. (Adam, $2005: 22$ ).

Sans vouloir tomber dans un conformisme pédagogique qui consiste à réfuter tout ce que la génération précédente a adulé, il nous semble difficile de concevoir la compatibilité entre l'approche par les compétences, placée au cœur de la Réforme algérienne et le mode d'analyse / catégorisation par type de texte qui se réduit le plus souvent dans les faits à une technique applicationniste et donc réductrice. Nous suivrons encore Adam dans cette voie: «l'on ne devrait parler ni de typologie de texte, ni de typologie de discours. Les typologies de discours doivent être remplacées par une réflexion sur les genres et la généricité » (ibid., 15).

\section{Formats pédagogiques et discursifs}

Corrélativement à ces réflexions sur texte et discours, en sus des questions de continuités linguistiques et curriculaires, les chercheurs estiment de plus en plus que c'est «le nouveau type de discours auquel l'étudiant est confronté à l'université qui fait problème, tant en compréhension qu'en expression» (Romainville, $2000: 39)$.Pollet (2001:26) parle d'une " rupture discursive qu'il est tout aussi indispensable de prendre en compte que les autres ruptures subies (psychologique, affective, sociale) ». À l'université algérienne, existent, comme en

Recherches en didactique des langues et des cultures, 13-1 | 2016 
France, différentes modalités d'enseignement: cours magistral, travaux dirigés (TD), travaux pratiques (TP), correspondant à différentes modalités de discours et de postures didactiques du côté des enseignants mais également à différentes modalités de réception et d'interaction du côté des étudiants. De ce point de vue, des travaux réalisés en Algérie qui permettent de comparer des cours enregistrés au lycée et à l'université, peuvent confirmer ces hypothèses. Dans les cours de lycée conduits par de jeunes enseignants engagés dans la Réforme, on peut observer un guidage de proximité des élèves qui semble assez proche des cours dialogués à la française :

ENS : mais // l'essentiel vous avez un texte / donc c'est un texte expositif que je peux résumer / parce qu'il a les ingrédients nécessaires pour faire son résumé /bien sûr // regardez / nous avons trois paragraphes / ces trois paragraphes forment les trois parties // que tout le monde connaît // oui / quelles sont les trois parties en général / nous avons une ///

EE : (réponse chorale des élèves) INTRODUCTION xxxxx

ENS : INTRODUCTION / s'il vous plaît / s'il vous plaît / un qui parle / ne parlez pas en groupe /

EE : DEVELOPPEMENT (réponse chorale des élèves) xxxxx

ENS : // donc en général nous avons une introduction / un développement / et UNE conclusion /// bon pour vous aider dans le travail / j'ai déjà fait un certain travail pour vous (l'enseignant montre du doigt ce qu'il a écrit au tableau) /

ENS : donc vous avez les parties entre crochets / vous avez là les différentes idées / du texte // ce sont les idées / j'ai pas dit les parties / j'ai // j'ai dit les différentes idées du texte dans ce tableau / bien sûr / avec les mots clés / [...]

On peut considérer que, dans ce cours dialogué, l'enseignant n'avancera dans le déroulement prévu que si les réponses des élèves sont effectivement les réponses attendues. Comme nous avons pu le montrer par ailleurs (Cortier, 2008) la parole de l'élève s'inscrit dans un texte déroulé par l'enseignant selon une progression bien orchestrée et, en règle générale, s'intégrant strictement dans les interstices de parole ouverts par l'enseignant. Les réponses chorales comme ci-dessus en sont une preuve.

Le guidage est ici maximal, puisque la préparation faite préalablement par l'enseignant, figure au tableau, étape par étape; l'enseignant montre la réalisation de la première étape de l'exercice (mots clés entre crochets).

De même, si l'on a pu observer dans certains cours des modalités pédagogiques peu usuelles à l'université: dictée de définitions ou de résumés et des formes de parler bilingue, passage de l'enseignement à l'arabe dialectal pour créer une connivence ou faire un peu d'humour, les cours magistraux universitaires se déroulent selon un format discursif relativement semblable aux cours français (Cf. Hachadi, 2006; Bouchard et al., 2005 ; Parpette, 2008 ; Cortier, Hachadi \& Amar Sharif, 2009), ce qui implique pour les étudiants des modalités de participation et d'implication relativement réduites en regard des cours du secondaire et une grande part accordée aux travaux personnels, également facteurs de déstabilisation.

Des propos d'étudiants relevés dans le corpus d'enquête confortent l'existence de ces ruptures linguistique, méthodologique et discursive pouvant aboutir à une surcharge cognitive et un découragement et confirmer les causes de l'échec important en première année universitaire. 


\section{Du côté des étudiants : profils d'entrée et représentations}

Les étudiants sont admis en Licence de français en fonction de la note obtenue au baccalauréat: en règle générale une note minimale de 12 est exigée. Les premiers résultats d'une étude en cours sur l'application de l'échelle des compétences du CECRL en Algérie et la passation de tests spécialement élaborés pour ce public (A. Ammouden, M. Ammouden, 2010) ainsi qu'une enquête à plusieurs volets, réalisée en 2010, montrent que cette note ne garantit pas l'acquisition des compétences nécessaires pour suivre ces études et qu'ils sont confrontés, pour certains durement, à cette rupture. Si les deux tiers des étudiants atteignent un niveau B1, près d'un tiers des étudiants inscrits en licence de français de Bejaia et Tizi Ouzou ${ }^{14}$, n'ont que le niveau $\mathrm{A}^{15}$, dans les compétences requises pour faire une licence de langue et littérature étrangère et ceci, à l'issue d'au moins dix ans d'enseignement / apprentissage du français. Un test réalisé à l'université d'Alger en $2010^{16}$ confirme des résultats insuffisants en expression écrite pour plus de $50 \%$ des étudiants, que ce soit pour la prise de notes ou les simples réponses à des questions de texte.

Comme le montrent certains témoignages, obtenus à partir de 89 rédactions d'étudiants de $1^{\text {ère }}$ année de licence ${ }^{17}$, ils sont nombreux à craindre les changements qu'impose ce passage à l'université, les propos de quelques-uns sont à interpréter comme de véritables «S.O.S» qui montrent que le linguistique, le discursif et le cognitif sont réellement indissociables:

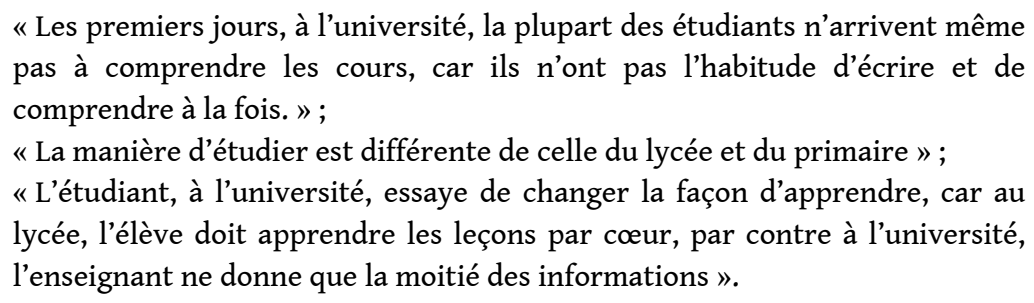

Si, avant l'université, "c'était l'enseignant qui faisait des recherches, préparait des cours » pour transmettre à l'étudiant "des tonnes d'informations (...) ce n'est pas le cas à l'université: l'étudiant doit travailler, faire des recherches, prendre des notes, exposer ... afin de réussir et surtout apprendre ». (Ammouden, 2012)

\section{Enquêtes réalisées auprès des étudiants}

\section{Conceptions et représentations de la langue française et rapport à l'écrit}

Les contenus des enseignements, aussi innovants et réfléchis soient-ils, ne peuvent améliorer la qualité des apprentissages que s'ils sont adaptés à la population étudiante concernée. Or, comme on a pu le souligner, la "culture écrite» des étudiants, leurs représentations des écrits réussis et leur rapport à la norme (Boch, Laborde-Milaa \& Reuter, 2004) sont nécessairement marqués par les acquis antérieurs des enseignements de l'arabe et des matières enseignées en arabe. Nous avons pu souligner plus haut la 
coexistence de cultures linguistiques et didactiques, qui, bien que partageant des traits communs, sont peu mises en convergence. Cette distance entre les enseignements et les savoirs acquis d'ordre linguistique, grammatical, langagier ne favorise guère la réussite des apprentissages linguistiques ou disciplinaires et peut au contraire agir négativement sur le rapport qu'entretiennent les étudiants avec les langues d'une part et l'écriture d'autre part, dans la mesure où ils sont accoutumés aux pratiques transmissives et à la tradition du Livre qui caractérisent les cours d'arabe. Jusqu'aux années 1980, l'enseignement est demeuré bilingue et les étudiants entrant à l'université étaient le plus souvent de "parfaits francophones» et de «mauvais » arabisants. Certains universitaires, parmi les plus anciens, ont figé leurs pratiques sur ce public et sont restés sur une conception de la langue française comme langue maternelle : on voit encore aujourd'hui, dans les nouveaux programmes de LMD, des traces bien vivantes de modules traditionnels peu compatibles avec les profils d'entrée des étudiants (d'après les tests de positionnement réalisés): modules d'histoire littéraire ${ }^{18}$ ou d'histoire de la langue, module "langues de spécialité » en première année. C'est pourquoi il nous a semblé utile d'interroger plus spécialement le rapport à l'écrit de ces étudiants, selon deux axes, celui des relations écrit / oral et celui du rapport à la norme, sachant que "l'apprentissage des contenus est indissociable du rapport à ces contenus que construit l'apprenant » et que celui-ci « conditionne en partie l'apprentissage de ces derniers » (Reuter et al. 2007 : 191)

33 L'enquête réalisée en 2010 se compose de questionnaires destinés aux étudiants de $1^{\text {ère }}, 2$ ème et $3^{\text {ème }}$ année et de productions écrites qui ont concerné ceux des deux premières années. Les premiers résultats sont fournis par un questionnaire distribué à 262 étudiants de licence de français, auxquels on demandait de réagir à des affirmations sur les relations écrit/oral.

L'affirmation « On dit facilement n'importe quoi, on écrit difficilement n'importe quoi » a été approuvée par 70,77\% pour les situations universitaires et 73,08\% pour les pratiques langagières de la vie quotidienne. L'affirmation « il vous est plus facile de comprendre l'oral que l'écrit» est approuvée par 83,59\% pour les pratiques de la situation universitaire et $88,17 \%$ pour celles de la vie quotidienne.

L'affirmation « Il vous est plus facile de parler le français que de l'écrire » a été également approuvée à 87,69 \% pour l'université et 91,22\% pour la vie quotidienne. Il ressort de ces résultats que le rapport qu'entretiennent les étudiants à l'écrit se caractérise par la difficulté de l'écrit face à l'oral.

\section{Analyse du corpus de productions écrites}

Le corpus d'analyse est constitué par la réunion des productions écrites de type " commentaire critique de citations» (portant sur les spécificités de l'écrit comparé à l'oral) soumises en examen universitaire à un total de 452 étudiants. L'ensemble a été préparé et analysé au moyen d'un logiciel d'analyses de données textuelles ${ }^{19}$ permettant d'obtenir des résultats quantitatifs et qualitatifs. Nous ne retiendrons ici que les résultats intéressant notre propos, soit le rapport à l'écrit et le statut de l'écrit en relation à l'oral, aux études et aux écrits universitaires.

Les énoncés recueillis permettent de montrer que l'écrit (en français) jouit d'un statut élevé chez les étudiants par rapport à l'oral mais que ce prestige va de pair avec les craintes qu'il suscite. Nous retrouvons dans les commentaires des étudiants des conceptions et représentations qui corroborent les analyses précédentes quant au 
rapport à la norme, à la prédominance de l'écrit par rapport à l'oral, confirmant la prégnance de la tradition culturelle et d'une survalorisation de l'écrit littéraire et du texte aux dépens des genres de l'oral et des formes de communication plus spécifiques.

Importance de l'écrit à l'université et dans la sphère académique

«L'importance de l'écrit dans le discours universitaire est essentielle par rapport à la langue orale »;

"A l'université, tout le travail des étudiants est basé sur l'écrit »;

"A l'université, les enseignants se basent sur l'écrit non pas sur l'oral, ils estiment le niveau d'un étudiant par rapport à son écrit personnel ».

\section{Difficultés de l'écrit en regard de l'oral : crainte de la faute et respect de règles} grammaticales

L'écrit se distingue de l'oral par « des règles très strictes. »;

« Il « s'attache à des normes et des lois obligatoires »;

« Il faut respecter toujours des règles », « surveiller la langue, les fautes »;

«A l'oral, les interlocuteurs ont tendance à négliger les règles de la grammaire et à s'exprimer à l'aide d'expressions agrammaticales mais cohérentes";

« On remarque qu'il y a des fautes qui se révèlent à l'écrit et non à l'oral ».

Importance des aspects graphiques et visuels : les fautes se voient

« Il est difficile d'écrire n'importe quoi car les fautes se voient (...).à l'oral il n'y a pas de trace »; "Pour arriver à communiquer par écrit il faut faire beaucoup d'efforts parce que oralement on peut dire tout ce qu'on veut (...) parce que on peut pas voir ce qu'on dit ».

\section{L'écrit fait peur}

"Cette forme de communication peut faire naitre parfois chez celui qui écrit une certaine forme d'anxiété, de stress, car elle laisse des traces, des souvenirs »; «La crainte de l'écriture et de la rédaction vient du fait qu'elle exige beaucoup trop de principe qu'on est obligé de prendre en considération (...),quand on demande à quelqu'un de nous rédiger quelque chose c'est directement la panique totale ».

\section{L'influence néfaste du parler et de l'oral sur l'écrit}

Le rapport à la norme, voire à une surnorme, que nous avons évoqué en relation avec les traditions linguistiques de l'arabe et du français apparaît très nettement lorsque les étudiants commentent l'influence néfaste du parler et de l'oral sur l'écrit et sur la qualité des discours universitaires.

- Le caractère libre et spontané de l'oral « affecte la qualité des discours universitaire "; "l'oral a beaucoup d'influence sur l'écrit parce que il y a des gens qui écrivent comme ils parlent, ils ne respectent pas les règles, ils [utilisent] le langage de la rue à l'université ». 
- L'utilisation des troncations (dites «abréviations" par certains) à l'oral et le langage familier sont considérés comme de sérieux problèmes qui affectent la qualité des discours universitaires :

" On emploie une langue vulgaire, des mots abrégés et des tronqués ";

«Ce langage familier entraine des influence sur l'écrit et sur le discours universitaire ";

«Si on écrit comme on parle on va détruire la langue et aussi il y aura des conséquences sur la qualité du discours»

- Le mélange de langues est également considéré comme préjudiciable à l'écrit alors qu'il constitue un habitus interactionnel :

"A l'oral le locuteur se permet de dire des mots, des phrases de mélanger entre les langues sans se soucier des règles grammaticales que l'écrit impose, ou de la cohérence, de l'enchainement. »

\section{L'écrit est un bien collectif}

« La langue écrite utilise des codes qu'un nombre important d'interlocuteurs comprend ";

«l'écrit c'est général, il est commun, il nous oblige à écrire de la même façon ";

« la langue est un fait social »;

« la langue est un phénomène social »;

« a langue et la parole ne constituent pas la même chose, car pour ce qui est de la langue, elle est collective, par exemple en France, les français utilisent tous la langue française mais contrairement à la parole qui, elle, est un acte individuel, chacun parle comme il veut, certains utilisent le français soutenu, certains utilisent le français courant et voire de nos jours presque toute cette nouvelle génération utilise le verlan ya pas de normes à suivre ou à respecter, chacun est libre de s'exprimer comme bon lui semble.»

39 Ces extraits montrent là encore l'influence des représentations liées à l'arabe sur les représentations de l'écrit et de l'oral en français. La situation de diglossie arabe littéral / standard vs dialectes qui se traduit toujours par des phénomènes d'hypercorrection, d'insécurité linguistique est ici bien visible et se trouve répercutée sur le français.

Mais l'écrit est aussi ce qui permet de manifester l'existence d'une communauté linguistique et, de ce fait, il permet de communiquer et de s'ouvrir à d'autres cultures :

«On trouve des difficultés pour comprendre un égyptien en parlant, car il utilise la langue arabe orale propre à son pays, bien que en écrivant on peut comprendre facilement ce qu'il veut dire. ";

"L'écrit n'a jamais perdu son importance (...) en ce qui concerne la transmission des informations et l'ouverture sur d'autres cultures. »

\section{L'écrit est l'apanage de l'élite et des intellectuels}

«Ce n'est pas donné à tout le monde de maitriser le domaine de l'écrit, il est souvent réservé à une certaine catégorie de gens "; «Ce n'est pas n'importe [qui] qui peut écrire»;

«la communication écrite peut être issue d'une société ou une catégorie intellectuel[le]»;

« Il demande la compétence de maitrise [ de la ]du langue, et l'expérience 
dans ce domaine comme: les écrivains, journaliste (littérature, et linguistique ...etc.) ».

\section{«Bien écrire est un art » (165)}

« L'écriture c'est un art à ceux qui la pratique[nt]»;

«L'écriture est un art, savoir écrire sans faute » (307);

"L'écriture est devenu un art (l'art de l'écrit »;

»L'écriture c'est un art et un don que peu de gens ont, car elle exigent

[exige] beaucoup de rigueur ";

«C'est ce qu'on voit dans les différents types de l'écrit, roman, récit, poésie etc. C'est l'art de transmettre des messages d'une façon attirante, agréable et belle »;

«L'écrit est un art peu facile à maitriser, car on ne peut pas écrire n'importe quoi. »

\section{Écrit, histoire, mémoire et religion : le Texte}

41 L'écrit ne protège pas seulement la langue mais aussi l'histoire et la culture $(140 ; 452)$ à travers les écrits des auteurs (260), la poésie, la civilisation et bien sûr la religion :

«L'écrit est un moyen (...) pour exprimer toutes les pensées et écrire les idées qu'un individu veut laisser pour ses descendants ainsi que écrire l'histoire" ;

«On peut écrire des lettres et des documents on les protège pour longtemps comme le cas chez les historiens et les poètes, etc. »;

« L'Histoire et les civilisations se transmettent avec l'écriture n'on [non] pas avec l'oral »;

" L'écrit utilisé depuis des siècles à [a] servi comme sauvegarde des savoirs et des textes de religion, des aides mémoire et beaucoup de chose avantageuses. ";

« Avec l'écrit, l'humain a pu connaitre ses origines, son dieu ».

En conclusion de cette dernière partie, nous pouvons sans doute faire l'hypothèse que l'influence de la tradition linguistique, grammaticale et littéraire de l'arabe et la non prise en compte des dimensions sociolinguistiques des langues, en termes d'évolutions, de contacts et de conflits, la dévalorisation de l'oral et des parlers et la surévaluation de l'écrit correspondante peuvent constituer un frein aux études universitaires, y compris dans les études de langue française, où cette tradition peut se voir confortée par les esprits conservateurs.

La méconnaissance de l'oral dans ses différentes dimensions et variations, apparaît nettement dans tous les commentaires des étudiants et doit inciter les linguistes et didacticiens universitaires à développer plus systématiquement des enseignements descriptifs et didactiques de l'oral, qu'il s'agisse des pratiques quotidiennes, médiatiques ou didactiques. On peut espérer obtenir alors une connaissance plus objective des discours écrits, de leur importance et de leurs spécificités. Cette dernière optique a été envisagée dans les unités fondamentales des masters FLE / FOS mis en place en 2010-2011 ${ }^{20}$, mais il serait souhaitable qu'une didactique de l'oral ou plutôt une sociodidactique de l'oral, basée sur des documents authentiques, présentant une variété de contextes francophones se constitue effectivement dès la première année universitaire, en dépit d'une apparente aisance des étudiants dans les interactions orales. 


\section{Conclusion} caractéristiques du contexte en didactique, aux différents niveaux macro, meso, micro, s'avère utile pour comprendre les contraintes institutionnelles et sociales qui pèsent sur les organisations et curricula, déterminante pour examiner les représentations et conceptions que les étudiants se font de la langue, à la fois vecteur et objet d'apprentissage et utile, sinon indispensable, pour la détermination des choix didactiques et des démarches à mettre en œuvre.

L'approche sociodidactique conjuguée à l'ingénierie de formation, à la méthodologie du travail universitaire et à une approche discursive liée aux genres textuels permet, au terme de ce parcours, de proposer un ensemble de recommandations à expérimenter pour faciliter l'acculturation universitaire des étudiants algériens :

- mieux connaitre les profils des étudiants, les soumettre à des tests de positionnement ou de niveau spécialement conçus en regard des exigences de la formation ;

- faire émerger les représentations des étudiants à propos de leurs pratiques langagières et de leur plurilinguisme (« biographies langagières »);

- travailler le rapport à l'écrit et les caractéristiques des «discours réussis », à partir des supports et des thématiques réellement rencontrées par les étudiants, mais ne pas négliger ni les pratiques langagières quotidiennes, ni les media, ni les textes littéraires ;

- privilégier le développement de compétences méthodologiques discursives aux dépens de la transmission de normes purement linguistiques ;

- développer les programmes et contenus des cours de méthodologie du travail universitaire et privilégier le développement de stratégies de lecture et d'écriture des genres universitaires en relation avec les « fonctions » que doit assumer l'étudiant ;

- initier progressivement à partir de la $3^{\mathrm{e}}$ année, les étudiants aux pratiques discursives qui caractérisent les discours scientifiques et l'écriture de la recherche.

On comprend que le développement de ces orientations et l'élévation du niveau de maitrise linguistique et discursive des étudiants ne pourra être atteint que par une politique nationale, dont on a pu voir récemment les prémices se mettre en place, grâce à une structuration du réseau des départements universitaires de français et aux travaux initiés au plan régional pour un réaménagement des curricula.

\section{BIBLIOGRAPHIE}

Adam J.-M. (2005). « La notion de typologie de textes en didactique du français : une notion

"dépassée" ?». Recherches, n² 42, pp. 11-23.

Ammouden, A. \& Ammouden, M. (2010). « Le français à l'université et l'échelle des compétences du Cadre Européen Commun de Référence pour les Langues ». Synergies Algérie, n 9, pp. 37-44. Disponible en ligne. http://gerflint.fr/Base/Algerie9/ammouden.pdf. 
Ammouden, M. (2012). L'apprentissage actif de l'écrit et /ou de l'oral en licence de français dans le cadre d'une approche intégrée. Thèse de doctorat de l'université de Bejaia.

Ammouden, M. \& Cortier, C. (2009). « L'enseignement du FLES et de l'arabe dans le contexte algérien : réflexions pour une didactique comparée à l'épreuve de la complexité et de l'interculturalité ». In Francia Leutenegger M-L., Schubauer-Leoni, et al. (éds) CDROM, Actes du 1er Colloque International de didactique comparée, 15-16 janvier 2009. Genève : Université de Genève \& ARCD.

Barré de Miniac, Ch. (2000). Le rapport à l'écriture : aspects théoriques et didactiques. Villeneuve d'Ascq : Presses universitaire du Septentrion.

Boch, F., Laborde-Milaa, I. \& Reuter Y. (eds). (2004). Les écrits universitaires. Pratiques, n - 121/122.

Bouchard, R. Parpette\& C. Pochard, J.C. (2005). « Le cours magistral et son double le polycopié : relations et problématiques de réception en $\mathrm{L} 2$ ». Cahiers du français contemporain, $\mathrm{n}^{\circ} 10$, Lyon : ENS LSH, 191-207.

Cortier C., (2008). La place de la parole des élèves dans les interactions de classe. Pour mieux comprendre l'émergence des formes d'indiscipline et d'incivilité », In Auger N., Moïse C. \& Romain-Schulz (éds), La violence verbale, Tome 2. Paris : L'Harmattan, 121-140.

Cortier C., Hachadi S., Amar Sharif Z., (2009). "Les cours magistraux dans les filières scientifiques des universités algériennes : caractéristiques discursives et interactionnelles« . In Defays, J-M \& Englebert, A. (dirs.). Principes et typologies des discours universitaires. Les discours universitaires : formes, pratiques, mutations, Tome I, Paris : L'Harmattan, 137-150.

Cortier, C., Kaaboub, (2010). «Le français dans l'enseignement universitaire algérien : enjeux linguistiques et didactiques ». In Parpette, C., Mangiante, J.M. Le Français dans le monde, Recherches et applications, $n^{\circ} 47,53-63$.

Coulon, A. 2005. Le métier d'étudiant : l'entrée dans la vie universitaire, $2^{\text {ème }}$ édition,.Paris : Economica.

Dabène, M.\& Reuter, Y., (1998). « Pratiques de l'écrit et modes d'accès au savoir dans l'enseignement supérieur ». Lidil, $n^{\circ} 17$.

Defays, J.M.\& Englebert, A., (2009). Principes et typologies des discours universitaires. Les discours universitaires : formes, pratiques, mutations, Tome I, Paris : L'Harmattan.

Delcambre I. \& Jovenet, A-M. (dirs), (2002). « Lire-écrire dans le supérieur ». Spirale, n²9, Disponible en ligne. http://spirale-edu-revue.fr/spip.php?article227.

Delcambre, I., Lahanier-Reuter, D., (2010). « Les Littéracies universitaires : Influence des disciplines et du niveau d'étude dans les pratiques de l'écrit ». Forumlecture.ch. http:// www.forumlecture.ch/sysModules/obxLeseforum/Artikel/431/Les-litteracies-universitaires.pdf.

De Ketele, J-M (2010). « La pédagogie universitaire : un courant en plein développement ». Revue française de pédagogie, $\mathrm{n}^{\circ} 172$, 5-13.

Fintz, C., (1998). La didactique du français dans l'enseignement supérieur. Paris : L'Harmattan.

Ghettas, C. (1995), « L'enfant algérien et l'apprentissage de la langue arabe à l'école fondamentale entre 5 et 9 ans ». Thèse de doctorat, Université Grenoble III.

Hachadi, S., (2006). « Les cours magistraux dans les filières scientifiques en Algérie : le cas de la biologie » . Mémoire de Magister en didactique du français de l'université de Blida. 
Parpette, C. \& Mangiante, J.M. (coord.) (2010). Faire des études supérieures en langue française. Le Français dans le monde, Recherches et applications, ${ }^{\circ} 47 .$, Paris : Clé international/FIPF.

Maurice, D., (2001). « Réussir la première année à l'université. La transition SecondaireUniversité : le projet Boussol ». Revue française de pédagogie, $\mathrm{n}^{\circ}$ 136, 77-86

Mangiante, J. M. \& Parpette, C., (2011). Le français sur objectifs universitaires. Grenoble : PUG.

Miled, M. (2005). « Vers une didactique intégrée : arabe langue maternelle et français langue seconde ». Le Français dans le monde, Recherches et applications, $n^{\circ}$ spécial. Paris : Clé international/ FIPF, 39-46.

Miled, M. (2008). « Identités linguistique et didactique convergente dans un contexte bilingue : l'exemple du français et de l'arabe au Maghreb ». In P. Martinez, D. Moore \& V. Spaëth, Plurilinguisme et enseignement : identités en construction. Paris : éd. Riveneuve, 197-203.

Moore, D., (2006). Plurilinguisme et école. Paris : Didier.

Parpette, C. (2008). « Les discours académiques oraux : évolution de représentations et des stratégies d'enseignement en FLE ». In Cortier, C., Bouchard, R., Le français dans le monde. Recherches et applications, $\mathrm{n}^{\circ} 43,114-126$.

Pollet, M.-C., (2001). Pour une didactique des discours universitaires. Bruxelles : De Boeck.

Reuter Y. (éd.), Cohen-Azria C., Daunay B., Delcambre I., Lahaner-Reuter D. (2007). Dictionnaire des concepts fondamentaux des didactiques. Bruxelles : De Boeck.

Romainville, M. (2000). L'échec dans l'université de masse. Paris : L'Harmattan.

Taleb-Ibrahimi, K., (1997). Les Algériens et leur(s) langue(s). Eléments pour une approche sociolinguistique de la société algérienne ( $2^{\mathrm{e}} \mathrm{ed}$.). Alger : Éditions El Hikma.

Taleb-Ibrahimi, K. (2007). «L'Algérie : coexistence et concurrence des langues », Actes du colloque Pour une histoire critique et citoyenne. Le cas de l'histoire franco-algérienne, 20-22 juin 2006, Lyon, ENS LSH. Disponible en ligne. http://colloque-algerie.ens-lsh.fr/communication.php3? id_article $=212$.

\section{NOTES}

1. Enquêtes de Hachadi Samir, Maitre-assistant à l'Université de Blida.

2. Ammouden, M. (2012) Thèse de doctorat Université de Bejaia \& Projet MeRSI AUF (2010-2013) coordonné par Carmen Stoean, Académie des Science économiques de Bucarest "Harmonisations des curricula de FLES/FOS dans les filières universitaires de français". Collaborations : Académie d'études économiques, Bucarest, ENS LSH Alger, Université Antonine-Beyrouth, Université Lyon 2, Université de Tirana, Albanie, Université Hassan II-Ain Chock, Casablanca.

3. Conférence au Séminaire "L'enseignement du français dans les pays de langue arabe", CIEP, 7 décembre 2010.

4. Soulignons que d'autres filières enseignent en français dans certaines universités sans que cela soit généralisé comme dans les disciplines scientifiques et médicales, par exemple la sociologie à Bejaia, l'économie à Bejaia et à Mascara, etc.

5. Conférence de Daniel Coste (avec K. Taleb-Ibrahimi), ENS de Lyon "Français et arabe. Deux langues en contact: divergence des contextes, convergence des approches? ", Séminaire "L'enseignement du français dans les pays de langue arabe", CIEP, 7 et 8 décembre 2010.

6. cf. le modèle constellation et gravitationnel de Abram de Swaan (1997) popularisé récemment par le baromètre des langues de Calvet, L.J.( http://wikilf.culture.fr/barometre2012/ ) qui 
différencie langues locales, centrales (nationale), supercentrales ou internationales (français, espagnol, arabe, hindi, etc.) et hypercentrales (anglais).

7. Ordonnance du 16 Avril 1976.

8. Les accords signés entre l'université de Blida et le Crapel de Nancy ont permis d'ouvrir dès 1993 une licence de FLE sur objectifs spécifiques, cf. Brakni \& Carton, (2010) Hommage à El Hocine Ghriss, Didacstyle, 3, 12-13.

9. Voir les travaux et manuels de Cherifa Ghettas, Professeur à l'université d'Alger, spécialiste de didactique de l'arabe.

10. Mémoire de Magister de Fatiha Ousseur : "Élaborer un journal lycéen selon la pédagogie de projet" Université de Blida, 2009 (Dir. C. Cortier)

11. Annaba, Bejaia, Oran.

12. Publications sous la direction de F. Sari, Professeur à l'Université d'Oran, avec l'appui du Service de coopération de l'Ambassade de France, Office des publications universitaires.

13. Corpus de Samir Hachadi, 2010. Thèse en cours: "L'influence du français langue d'enseignement sur les résultats des étudiants en première année universitaire. Cas de la faculté des sciences agrovétérinaires et biologiques de l'université de Blida".

Convention de transcription : / (pause courte), // pause longue, XXXX(inaudible) INTRODUCTION (voix chorales)

14. En Kabylie, région réputée particulièrement francophone et francophile, dans deux universités où l'accès à cette formation est pourtant particulièrement réservé à ceux qui obtiennent de très bonnes notes en français au Bac.

15. On estime qu'un niveau B1 voire B2 est nécessaire pour suivre un cursus universitaire.

16. Test conçu par une stagiaire FLE à l'université d'Alger 2 pour les étudiants de première année de licence de l'année 2010-2011.

17. Corpus de M. Ammouden (2012)

18. Panorama de la littérature Française II : La littérature française du 10ème siècle et 20ème siècle :- Moyen âge (10ème, 15ème siècle) ; - Renaissance (16ème siècle) ; - 17ème siècle ;- 18ème siècle - 19ème siècle - 20ème siècle

19. Logiciel Alceste, créé par M. Reinert, diffusé par la société Image, Toulouse : www.imagezafar.com/index_alceste.htm

20. Masters professionnalisant à recrutement national ouvert à Alger, Constantine et Mostaganem avec le concours des services de coopération.

\section{RÉSUMÉS}

En raison des particularités du contexte algérien et du changement de langue d'enseignement entre l'enseignement secondaire et l'université, le passage d'un niveau à l'autre provoque une rupture discursive et méthodologique qui se traduit par un taux d'échec important en $1{ }^{\text {ère }}$ année. Pour faciliter cette transition, qui nécessite une «affiliation intellectuelle » des étudiants, une étude comparant les caractéristiques des discours pré-universitaires et universitaires, identifiant précisément leurs difficultés, ainsi que leurs représentations et relations à l'écrits est apparue nécessaire, afin de préparer cette acculturation aux discours universitaires et de relier programmes académiques et études des genres textuels. 
For several reasons specific to the Algerian context, including the transition from Arabic to French language of instruction, the university entrance leads to a methodological break, discursive, linguistic and cognitive. Facilitating this transition requires an "intellectual affiliation". That would, among other things, aim to compare the characteristics of preuniversity and university speech, to identify students' problems, as well as their representations and relationship to writing; the overall aim being to consider possible pathways that would facilitate the acculturation to academic discourse. By reference to the genres, this article allows to link academic programs and disciplinary practices, with the aim to renew curricula.

\section{INDEX}

Mots-clés : Algérie, transition académique, licence, discours universitaires, rapport à l'écrit, genres textuels

Thèmes : Varia

Keywords : Algeria, undergrad, academic transition, academic literacies, relationship to writing, textual genres

\section{AUTEURS}

\section{M'HAND AMMOUDEN}

Laboratoire LAILEMM ; Université de Bejaia

M'Hand Ammouden est maître de conférences HDR en didactique des langues à l'université Abderrahmane Mira de Bejaia et directeur du laboratoire de recherche/formation en Langues Appliquées et Ingénierie des Langues en Milieu Multilingue (LAILEMM). Ses recherches et publications, qui portent essentiellement sur les approches intégrées des compétences de l'oral et/ou de l'écrit, sur les genres textuels ordinaires et sur le plurilinguisme, s'inscrivent dans le cadre de la sociodidactique.

Courriel : m.ammouden[at]yahoo.fr

\section{CLAUDE CORTIER}

UMR ICAR 5195 ; Université de Lyon

Claude Cortier est enseignante et chercheure en sociolinguistique et didactique des langues (UMR CNRS 5191 ICAR, Université de Lyon), membre de plusieurs réseaux scientifiques internationaux. Dans le cadre d'une approche sociodidactique, ses travaux portent sur l'histoire du FLES, l'idéologie et les représentations des langues-cultures, les approches interlinguistiques et interculturelles en situations minoritaires ou migratoires, le français langue d'enseignement et de scolarisation et l'analyse des discours et interactions didactiques en contextes plurilingues. Courriel : claude.cortier[at]gmail.com 